\title{
Significance-Driven Graph Clustering ${ }^{\star}$
}

\author{
Marco Gaertler, Robert Görke, and Dorothea Wagner \\ Faculty of Informatics, Universität Karlsruhe (TH) \\ \{gaertler, rgoerke, wagner\}@informatik. uni-karlsruhe.de
}

\begin{abstract}
Modularity, the recently defined quality measure for clusterings, has attained instant popularity in the fields of social and natural sciences. We revisit the rationale behind the definition of modularity and explore the founding paradigm. This paradigm is based on the trade-off between the achieved quality and the expected quality of a clustering with respect to networks with similar intrinsic structure. We experimentally evaluate realizations of this paradigm systematically, including modularity, and describe efficient algorithms for their optimization. We confirm the feasibility of the resulting generality by a first systematic analysis of the behavior of these realizations on both artificial and on real-world data, arriving at remarkably good results of community detection.
\end{abstract}

\section{Introduction}

Discovering natural groups and large scale inhomogeneities is a crucial task in the exploration and analysis of large and complex networks. This task is usually realized with clustering methods. The majority of algorithms for graph clustering are based on the paradigm of intra-cluster density versus inter-cluster sparsity. Several formalizations have been proposed and evaluated, an overview of such techniques is given in [1]. Recently, Girvan and Newman 2] proposed the objective function modularity, which indirectly incorporates this paradigm. Modularity evaluates a clustering based on the fraction of intra-cluster edges compared to the expected value of this number. Modularity was first introduced as a quality measure of a clustering, however, a simple greedy algorithm, solely based on modularity, has been proposed shortly after by Newman [3]. The definition of modularity and this algorithm evoked a surge of interest, yielding many studies concerning different applications, such as protein interaction dependencies, recommendation systems or social network analysis, and possible adjustments (see e.g. 45667]). Moreover, a range of alternative algorithmic approaches has been proposed, based on a greedy agglomeration [8], on spectral division [1011, simulated annealing [1213] and extremal optimization [14]. Recently, it has been proven, that it is $\mathcal{N} \mathcal{P}$-hard to optimize modularity [15],

\footnotetext{
* This work was partially supported by the DFG under grants WA 654/14-3 and by EU under grant DELIS (contract no. 001907) and CREEN project (contract no. 012684).
} 
which justifies the need for heuristics and approximations. Note that little is known on the approximability of modularity.

However, to our knowledge, no systematic evaluation of modularity-based clustering has been performed, yet. In this work, we conduct such an evaluation and, additionally, we advance towards a profound understanding of the rationales modularity is based upon. We formally state and investigate the generalized founding paradigm for the significance of a clustering as the trade-off between the achieved quality and the expected quality for random networks incorporating the intrinsic properties of the original network. We experimentally evaluate realizations of this paradigm (including modularity itself) and extensively evaluate algorithms that each optimize a realization, followed by a partial discussion of their behavior. Furthermore, we present an algorithm that efficiently optimizes the particularly promising realization relative performance significance in $O\left(n^{2} \log n\right)$ time. We compare the performance of these algorithms in terms of clustering quality to that of other clustering algorithms, on a set of random pre-clustered graphs and complement our findings with results on real data. Our results indicate the feasibility of the paradigm in that, on the whole, our algorithms surpass the benchmark algorithms, and in that the generality of the approach is justified by specific realizations excelling on real-world data.

This paper is organized as follows: After introducing the necessary preliminaries for graph clustering and some quality measures (Sec. 2), we give the formal definition of our significance paradigm and present some realizations (Sec. 3). Section 4 scrutinizes the greedy algorithms which are employed to obtain clusterings with high significance score, including an efficient implementation for a quick divisive merge. The setup and the results of the experimental evaluation are described in Section 5 which are followed by a conclusion.

\section{Preliminaries}

Throughout this paper, we will use the notation of [1]. More precisely, we assume that $G=(V, E, \omega)$ is an undirected, weighted, and simple graph and $\omega: E \rightarrow[0,1]$. For a node $v$, we define the node weight $\omega(v)$ as the sum of the weight of its incident edges. Let $|V|=: n,|E|=: m$ and $\mathcal{C}=\left\{C_{1}, \ldots, C_{k}\right\}$ a partition of $V$. We call $\mathcal{C}$ a clustering of $G$ and the $C_{i}$ clusters; $\mathcal{C}$ is called trivial if either $k=1$, or all clusters $C_{i}$ contain only one element. In the following, we often identify a cluster $C_{i}$ with the induced subgraph of $G$, i. e., the graph $G\left[C_{i}\right]:=\left(C_{i}, E\left(C_{i}\right), \omega_{\mid E\left(C_{i}\right)}\right)$, where $E\left(C_{i}\right):=\left\{\{v, w\} \in E: v, w \in C_{i}\right\}$. Then $E(\mathcal{C}):=\bigcup_{i=1}^{k} E\left(C_{i}\right)$ is the set of intra-cluster edges and $E \backslash E(\mathcal{C})$ the set of inter-cluster edges. The set $E\left(C_{i}, C_{j}\right)$ denotes the set of edges connecting nodes in $C_{i}$ to nodes in $C_{j}$. The number of intra-cluster edges is denoted by $m(\mathcal{C})$ and the number of inter-cluster edges by $\bar{m}(\mathcal{C})$. We denote the number of nonadjacent pairs of nodes that are in the same cluster as $m(\mathcal{C})^{c}$, and the number of 
non-connected pairs of nodes that are not in the same cluster as $\bar{m}(\mathcal{C})^{c}$. Modularity is defined ([9]) as:

$$
\bmod (\mathcal{C}):=\frac{m(\mathcal{C})}{m}-\frac{1}{4 m^{2}} \sum_{C \in \mathcal{C}}\left(\sum_{v \in C} \operatorname{deg}(v)\right)^{2}
$$

We measured the quality of clusterings with a range of of quality indices, discussed e.g., in [1], however, we set our focus on the indices coverage ([1]) and performance ([16]) in this work, since they are the most well studied ones. In brief, coverage is the fraction of intra-cluster edges and performance is the fraction of correctly classified node pairs. For a discussion of these indices we refer the reader to the given references, and simply state their formal definitions in Equations (2) and (3):

$$
\operatorname{cov}(\mathcal{C}):=\frac{m(\mathcal{C})}{m}=\frac{m(\mathcal{C})}{m(\mathcal{C})+\bar{m}(\mathcal{C})}
$$

$$
\operatorname{perf}(\mathcal{C}):=\frac{m(\mathcal{C})+\bar{m}(\mathcal{C})^{c}}{\frac{1}{2} n(n-1)}
$$

The fact that modularity can be expressed as coverage minus the expected value of coverage (see Sec. 3.1 and [9]) motivates the general paradigm we state in the next section. These definitions generalize in a natural way as to take edge weights into account. Thus, $\omega(\mathcal{C})(\bar{\omega}(\mathcal{C}))$ denotes the sum of the weights of all intra-cluster (inter-cluster) edges and $W$ denotes the sum of all edge weights. In the terms $\omega(\mathcal{C})^{c}$ and $\bar{\omega}(\mathcal{C})^{c}$ the weight between a non-adjacent pair of nodes is set to the maximum edge weight in $E$ and the weight between adjacent nodes is the difference of the maximum edge weight and the weight of the edge between them.

\section{The Significance Paradigm}

In the significance paradigm a good clustering is characterized by having a high quality compared to the value obtained for a random network that reflects specific structural properties that are expected to be present in the graph, as predefined in an appropriate null hypothesis. The structural properties of a graph can include characteristics such as the sequence of degrees, the number of nodes, the clustering coefficient, the degree distribution etc. These properties need not determine a graph completely but define a family of graphs incorporating them. A configuration is a specific realization of these properties, i.e., a specific graph. Every realization of the significance paradigm requires a quality measure, a null hypothesis, and a mode of comparison of both. Since measuring the quality of a clustering is a well-studied field, we present a way to extend a given quality index $\mathcal{M}$ to our paradigm. As, in this context, modularity (1) extends coverage (2), the concept of significance is a true generalization of modularity.

Definition 1. Given a quality index $\mathcal{M}$ and a clustering $\mathcal{C}$, we define the significance $S_{\mathcal{M}}^{\odot}$ of a clustering $\mathcal{C}$ as the corresponding quality index respecting our paradigm in the following way: 


$$
S_{\mathcal{M}}^{\odot}(\mathcal{C}):=\mathcal{M}(\mathcal{C}) \odot \mathbb{E}_{\Omega}[\mathcal{M}(\mathcal{C})],
$$

where $\mathbb{E}_{\Omega}[\mathcal{M}]$ is the expected value of the quality index $\mathcal{M}$ for the clustering $\mathcal{C}$ with respect to a suitable probability space $\Omega$ and $\odot$ is a binary operator on real numbers.

The following example illustrates the intention of this definition. Although, many quality measures $\mathcal{M}$ are normalized to the interval $[0,1]$, it is often hard to associate a specific value with a meaningful interpretation as showcased in Figures 1 and 2] This can be an intrinsic pattern of the measure and not merely

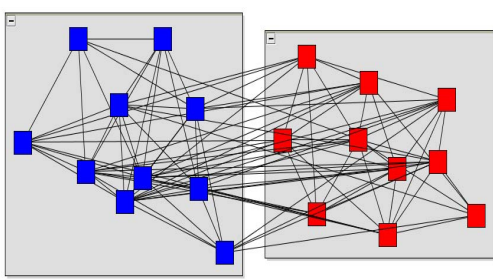

Fig. 1. A random split of a $G(n, p)$ with $n=20$ and $p=0.5$, where coverage yields a value of 0.66

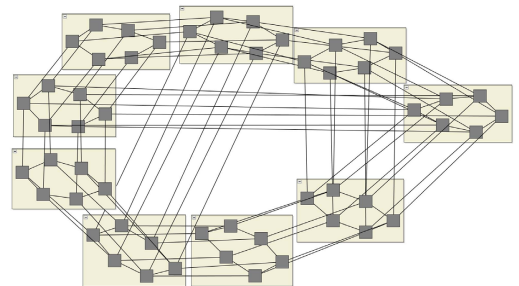

Fig. 2. A meaningful clustering of an interconnected, six-sided tube, yielding a coverage of 0.43

an artifact of the normalization. However, the comparison of the achieved quality with the expected quality (with respect to a suitable model) intuitively yields a 'relative' variant of the measure. Thus, it potentially allows for a better interpretation of the scale (modularity value for Fig. 1] 0.128, for Fig. 2, 0.316).

\subsection{Probability Space}

In the following we briefly discuss a suitable probability space $\Omega$ required for Definition 1, which we use throughout this paper. The importance of the structural properties of a graph may differ depending on the application, we restrict ourselves to a basic setup, where the degrees of the nodes are considered to be a defining property. Thus $\Omega$ consists of the family of all graphs having the same sequence of degrees as the input graph, with uniform probability.

However, even for simple quality indices, such as coverage, we are not aware of a closed formulation for the expectation. Instead of designing random processes to obtain expectation values, we consider a heuristic that was already used in 9 . The heuristic assigns to each edge $e=\{v, w\}$ a probability of $\operatorname{deg}(v) \operatorname{deg}(w) / 2 m$ in the unweighted case, and an expected weight of $\omega(v) \omega(w) / 2 W$ in the weighted case. While this heuristic does not rigorously respect the expected sum of all node weights, the error term tends to zero for graphs with a sublinear maximum degree, see Appendix A A degenerate example that clearly illustrates this discrepancy is the unweighted, complete graph $K_{n}$. For this graph, the original probability space contains only one element, thus yielding $\mathcal{M}(\mathcal{C})=\mathbb{E}_{\Omega}[\mathcal{M}(\mathcal{C})]$, which is not true for the heuristic. 
The concept of significance is related to the notion of $p$-values in statistical hypothesis testing. The $p$-value of a value $t$ observed for a random variable $T$ is the probability that under the assumption of a given null hypothesis, $T$ assumes a value at least as unfavorable to the null hypothesis as the observed value $t$. In general, the null hypothesis is rejected, if the $p$-value is smaller than the statistical significance level (see e.g. [17]). However, in our concept we do not reject a null hypothesis, which we assume to reasonably describe observed graphs. Instead, we compare the achieved quality of a clustering to the expected value, in order to judge its relevance.

\subsection{Implementations of the Significance Paradigm}

The heuristic presented above enables us to study four implementations of the significance paradigm, namely, coverage and performance as quality indices and subtraction and division as the binary operators. Using coverage and subtraction, modularity is one of the implementations. Table 3.2 summarizes the formulas of the resulting four implementations of the significance paradigm. For a discussion of performance in weighted graphs see [1], the proof for $\mathbb{E}[$ performance $]$ can be found in the Appendix $\mathrm{A}$

Table 1. Quality indices and their expected values ( $\omega_{\max }$ : maximum edge weight)

\begin{tabular}{|c|c|c|}
\hline measure & coverage & performance \\
\hline $\mathcal{M}$ & $\frac{m(\mathcal{C})}{m}$ & $\frac{m(\mathcal{C})+\bar{m}(\mathcal{C})^{c}}{0.5 \cdot n(n-1)}$ \\
\hline $\mathbb{E}[\mathcal{M}]$ & $\sum_{C \in \mathcal{C}}\left(\frac{\sum_{v \in C} \operatorname{deg}(v)}{2 m}\right)^{2}$ & $\frac{\sum_{C \in \mathcal{C}}\left(\left(\sum_{v \in C} \operatorname{deg}(v)\right)^{2} / m-\left(\sum_{v \in C} 1\right)^{2}\right)+n^{2}-2 m}{n(n-1)}$ \\
\hline $\mathcal{M}_{\text {weighted }}$ & $\frac{\omega(\mathcal{C})}{W}$ & $\frac{\omega(\mathcal{C})+\omega_{\max } \bar{m}(\mathcal{C})^{c}+\left(\omega_{\max } \bar{m}(\mathcal{C})-\bar{\omega}(\mathcal{C})\right)}{0.5 \cdot n(n-1) \cdot \omega_{\max }}$ \\
\hline $\mathbb{E}\left[\mathcal{M}_{\text {weighted }}\right]$ & $\sum_{C \in \mathcal{C}}\left(\frac{\sum_{v \in C} \omega(v)}{2 W}\right)^{2}$ & $\frac{\sum_{C \in \mathcal{C}}\left(\sum_{v \in C} \omega(v)\right)^{2} / W+\omega_{\max }\left(n^{2}-\sum_{C \in \mathcal{C}}|C|^{2}\right)-2 W}{n(n-1) \omega_{\max }}$ \\
\hline
\end{tabular}

Note that the weighted versions of modularity are true generalizations of the unweighted case, since setting each weight to 1 yields the unweighted formulas. Thus, we restrict our analyses on the weighted case. The weighted variant of modularity has also been defined by Newman in [18. Based on Table 3.2 we now define the following implementations, with a nomenclature borrowed from approximation theroy:

$$
\begin{aligned}
& \mathrm{S}_{\text {cov }}^{-}:=\text {coverage }-\mathbb{E}[\text { coverage }] \quad \text { (equals modularity) } \quad \mathrm{S}_{\text {cov }}:=\frac{\text { coverage }}{\mathbb{E}[\text { coverage }]} \\
& \underbrace{\mathrm{S}_{\text {perf }}^{-}:=\text {performance }-\mathbb{E}[\text { performance }]} \\
& \text { absolute variants (subtractive) } \\
& \underbrace{\mathrm{S}_{\text {perf }}^{\circ}:=\frac{\text { performance }}{\mathbb{E}[\text { performance }]}}_{\text {relative variants (divisive) }}
\end{aligned}
$$


As we shall see in the evaluation in Section [5] these implementations differ significantly in their behavior, although they are all derived from the same paradigm.

\section{Significance-Clustering Algorithms}

In this section, we describe the algorithms that are used to obtain clusterings with high values of significance. As suggested by Newman in 3] we employ a straightforward greedy heuristic. This allows for a fair evaluation for all the variants of significance introduced in Section 3.2. The usage of a heuristic or an approximation is strongly encouraged by the fact that an $\mathcal{N} \mathcal{P}$-completeness proof of this optimization problem has recently been presented in [15].

\subsection{The Greedy Algorithm}

For a given significance measure $S$ the greedy algorithm starts with the singleton clustering and iteratively merges those two clusters that yield largest increase or the smallest decrease in significance. After $n-1$ merges the clustering that achieved the highest significance is returned. The algorithm maintains a symmetric matrix $\Delta S$ with entries $\Delta S_{i, j}$ equaling $S\left(\mathcal{C}_{i, j}\right)-S(\mathcal{C})$, where $\mathcal{C}$ is the current clustering and $\mathcal{C}_{i, j}$ is obtained from $\mathcal{C}$ by merging clusters $C_{i}$ and $C_{j}$. The pseudo-code for the greedy algorithm is given in Algorithm 1 .

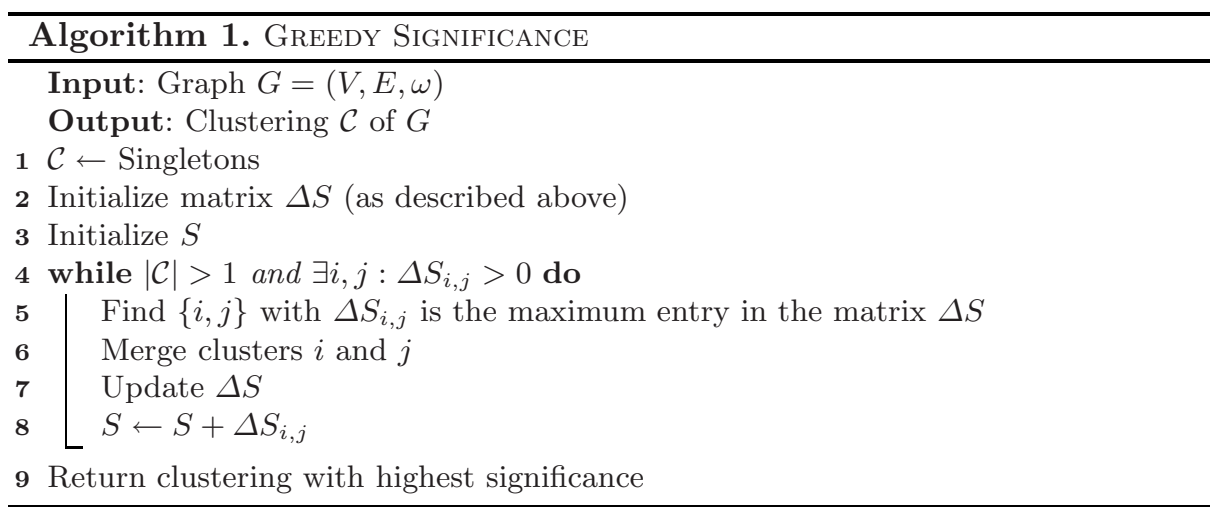

\subsection{Runtime Analysis}

Due to the special structure of the significance measures introduced in Section 3.2, a precise analysis can be given. We define the matrices $\Delta \mathcal{M}$ and $\Delta \mathbb{E}[\mathcal{M}]$ analogously to $\Delta S$. First, note that coverage and performance can be updated locally. More precisely, let $\mathcal{C}$ be a clustering, then $\operatorname{cov}\left(\mathcal{C}_{i, j}\right)$ can be computed using $\operatorname{cov}(\mathcal{C}), E\left(C_{i}\right), E\left(C_{j}\right)$ and $E\left(C_{i}, C_{j}\right)$. The same holds for performance and for both values of expectation. Thus, we obtain for the absolute variants the update formula: $\Delta S_{k,(i j)}=\Delta \mathcal{M}_{i, k}+\Delta \mathcal{M}_{j, k}-\Delta \mathbb{E}\left[\mathcal{M}_{k, i}\right]-\Delta \mathbb{E}\left[\mathcal{M}_{k, j}\right]$, where $C_{(i j)}$ 
corresponds to the merged cluster of $C_{i}$ and $C_{j}$; see [19] for details. Due to this locality, only two rows and columns are affected, namely the $i$ th and the $j$ th, requiring $O(n \log n)$ update time, using heaps to store the rows. The heaps help to quickly find the maximum entry in $\Delta S$, i.e. the best improvement. However, for the relative variants, the whole matrix has to be updated, yielding at least a quadratic runtime for a standard implementation. In Section 4.3, we present a faster method using kinetic heaps and geometry.

Employing a simple data structure for clusterings e.g. representing clusters by linked lists and storing a collection of pointers to their head, one observes that Step 1, Step 3 and Step 9 run in $O(n)$ time. The matrix $\Delta S$ is initialized in $O\left(n^{2}\right)$ time. The loop at Step 4 is executed $n-1$ times. Step 5 runs in $O(n)$ time, since the rows of $\Delta S$ are stored as heaps. The merge of two clusters (step 6) and the update of $S$ (step 8) require at most linear time. Thus, the total runtime is dominated by the the time required for updating $\Delta S$, which yields the following lemma:

Lemma 1. Algorithm 1 runs in $O\left(n^{2} \log n\right)$ time for the absolute variants.

For the relative variants, an analog to Lemma 1 yields a runtime of $O\left(n^{3}\right)$, however, in Lemma 2 we improve this upper bound for relative variants employing Algorithm 2] It is not hard to see that the first local optimum of $S$, that the absolute greedy heuristic attains, is its global optimum, since then the matrix $\Delta S$ is non-positive, allowing no further increase in $S$ with any future merge. This is due to the linear update of $\Delta S$ that consists of simply adding up specific entries, thus keeping the matrix non-positive after future merges. This can result in a substantial decrease in running time if the graph is known to have a fine clustering structure, i.e., if $|\mathcal{C}| \in \omega(1)$ (if the number of clusters is dependent on $n$ ). The loop at Step 4 can then be terminated after $o(n)$ merges. A similar observation holds for the relative variants, as described in the next section.

\subsection{Quick Divisive Merge}

Considering the relative variants, after a merge, both $\mathcal{M}$ and $\mathbb{E}[\mathcal{M}]$ change, and thus all values of $\Delta S_{k, l}$ have to be recomputed after each merge, even though $\Delta \mathcal{M}_{k, l}$ and $\Delta \mathbb{E}[\mathcal{M}]_{k, l}$ might not change. The update of $\Delta S$ thus requires $O\left(n^{2}\right)$ time. In Algorithm 2 we show how this update can be performed in $O(n \log n)$ time using a geometric embedding of $\Delta S$. The algorithm uses the fact that if we assign to each entry $\{i, j\}$ of $\Delta S$ a point $p_{i j}=\left(\mathcal{M}\left(\mathcal{C}_{i, j}\right), \mathbb{E}\left[\mathcal{M}\left(\mathcal{C}_{i, j}\right)\right]\right)=$ $\left(\mathcal{M}+\Delta \mathcal{M}_{i j}, \mathbb{E}[\mathcal{M}]+\Delta \mathbb{E}[\mathcal{M}]_{i j}\right)$, the best merge corresponds to the point $p_{\max }$ that maximizes $y(p) / x(p)$. Since we only regard increases, and $\Delta \mathbb{E}[\mathcal{M}]_{i j} \geq 0$, we only need to consider the points in quadrant one. The point $p_{\max }$ can be found by a tangent query through 0 . Thus $p_{\max }$ lies on the convex hull of this set of points, unless we encounter the degenerate case, where $x(p)=0$, which can easily be detected and handled. After a merge, the points that correspond to rows or columns $k$ or $l$, are updated as sketched out in Section 4.2. Then, all points are implicitly shifted with respect to the new values of $\mathcal{M}$ and $\mathbb{E}[\mathcal{M}]$ in line 3. which means that $R$ is initialized as 0 before the first merge. 
In terms of running time, the crucial steps in Algorithm 2 are lines 1 and 2. Brodal and Jacob 20] introduce kinetic heaps using space only linear in the number of points stored, that support the tangent queries in line 1 in $O(\log n)$ time and insertions and deletions in $O(\log n)$ amortized time per operation. This yields a total running time of $O(n \log n)$ per merge of clusters, since only points assigned to entries of $\Delta S$ that correspond to clusters $k$ or $l$ have to be recomputed. The update of the values $\mathcal{M}$ and $\mathbb{E}[\mathcal{M}]$ is realized by simply shifting the origin accordingly, saving $\Omega\left(n^{2}\right)$ updates of points. Thus, we arrive at the following:

Lemma 2. By employing quick divisive merge (Algorithm 2), Algorithm 1 runs in $O\left(n^{2} \log n\right)$ time for the relative variants.

Similar to the absolute variants, the relative heuristic finds a single optimum. The update of $S$ and of the entries of $\Delta S$ corresponds to a vector addition. The greedy strategy chooses the update that results in the best increase in $S$. If at any time $S$ cannot be increased, then no entry of $\Delta S$ yields a greater gradient in the plane than $S$. But then in turn, no vector addition can ever produce an updated entry of $\Delta S$ that has a higher gradient than that of $S$. Thus, the first peak of $S$ that the relative algorithm finds is the maximum of the algorithm. It can even be observed that there are no further local optima, as the algorithm always chooses the most gentle decrease in $S$.

Note that the above observations and lemmas generalize to all implementations of significance, such as relative performance significance, where a merge of two clusters entails an addition of corresponding entries of $\Delta S$ (or of $\Delta \mathcal{M}_{w}$ and $\left.\Delta \mathbb{E}\left[\mathcal{M}_{w}\right]\right)$. However, special attention has to be paid to implementations, where points in quadrant three can also lead to an increase. Then, two tangent queries have to be performed and the update has to be adapted.

If a graph is known to have a fine community structure (e.g. $\Omega(\sqrt{n})$ communities), the running time of Algorithm 1 decreases accordingly, if it is reasonable to assume that the algorithm identifies the community structure.

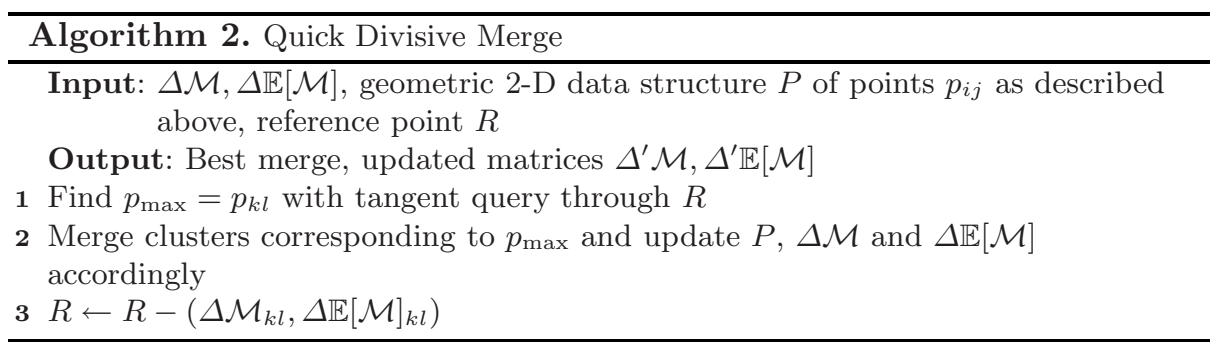

\section{Evaluation}

In the following we first describe the general model used to generate the instances for the experimental evaluation, then we present and discuss the results. Our setup for the evaluation is an adaption of the benchmark presented in [21]. 


\subsection{Random Uniform Clustered Graphs}

We use a random partition generator $\mathcal{P}(n, s, v)$ that creates a partition $\left(P_{1}, \ldots, P_{k}\right)$ of $\{1, \ldots, n\}$ with $\left|P_{i}\right|$ being a normal random variable with expected value $s$ and standard deviation $s / v$. Note that $k$ depends on the choice of $n, s$ and $v$, and that the last element $\left|P_{k}\right|$ of $\mathcal{P}(n, s, v)$ is possibly significantly smaller than the others. In order to settle this, we relaxed the size constraint i.e., if the last cluster size variable $\left|P_{k}\right|$ is too small or too large but the number of unassigned or additional nodes is less than one third of the expected cluster size, we add or delete the corresponding nodes. However, if the gap exceeds one third, we reject the partition and generate a new one. This may bias the generation process, yet we observed only few rejections during our experiments.

Given a partition $\mathcal{P}(n, s, v)$ and probabilities $p_{\text {in }}$ and $p_{\text {out }}$, a uniformly random clustered graph $(G, \mathcal{C}, \omega)$ is generated by inserting intra-cluster edges with probability $p_{\text {in }}$ and inter-cluster edges with probability $p_{\text {out }}$. In case a graph generated that way is not connected, additional edges combining the components are added. Edge weights $\omega$ are then set as to reflect the given partitioning. A weight from $\left[0, p_{\text {out }}\right]$ for each inter-cluster edge and from $\left[p_{\text {in }}, 1\right]$ each for intra-cluster edge is uniformly at random selected and assigned.

For our experiments we considered the following values of $p_{\text {in }}, p_{\text {out }}$ and $(n, s, v)$. We set $v=4$ and choose $s$ uniformly at random from $\left\{\frac{n}{\ell} \mid 2 \leq \ell \leq \sqrt{n}\right\}$. For $n=100$ and $n=1000$ we consider all combinations of $p_{\text {in }}$ and $p_{\text {out }}$ at a distance of 0.05. Among these we roughly refer to combinations supporting dense, sparse, strong and random community structure by $A_{\text {dense, }}, A_{\text {sparse }}, A_{\text {strong }}$ and $A_{\text {rand. }}$, respectively, as sketched out in Figure 3. For each combination and each algorithm, experiments were repeated until statistical significance has been attained with an $\alpha$ -

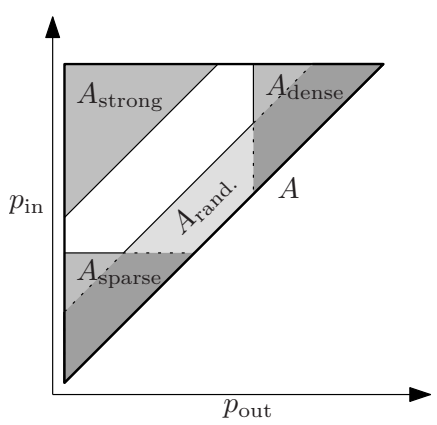

Fig. 3. Combinations of $p_{\text {in }}$ and $p_{\text {out }}$ level of 0.95 and a confidence interval of length 0.1 for each quality index measured. With an average of 223 runs per experiment we conducted about one million runs.

\subsection{Evaluation Process}

We conduct a qualitative comparison of the four implementations of the significance paradigm, as described in Section 3.2. The corresponding clusterings with high significance scores are obtained with the greedy heuristic based on Algorithm 1. Similar to the benchmark presented in [21, we restrict ourselves to qualitative aspects of the resulting clustering, and basic structural aspects, such as the number of clusters. Although this is only peripheral to our work, 
the effective runtimes of our basic and non-optimized Java 1.5 implementations ranged from a few milliseconds for 100 nodes using absolute variants to several minutes for 1000 nodes using relative variants, on an AMD Opteron $2.2 \mathrm{GHz}$ machine.

\subsection{Computational Results}

Experiments. As a reference to the benchmark, we compare our findings to an established clustering algorithm, Markov Clustering (MCL) [16], The results of the experiments with respect to performance and coverage are given in Figure 4 and 5 respectively. We omit further plots illustrating the results on other benchmark algorithms (e.g. GMC [1]), other quality indices (e.g. inter-cluster conductance, see 21]) and structural observations due to space limitations.

At a first glance, the statistical results of the two absolute variants $\left(\mathrm{S}_{\mathrm{cov}}^{-}\right.$and $\left.\mathrm{S}_{\text {perf }}^{-}\right)$strongly resemble each other, see Figure 4(c) and 4(d). For example their achieved quality does not differ by more than $2.6 \%$ with respect to performance, see Figure 4 Similar observations hold for the number of clusters. However, the relative variants $\left(S_{\dot{\mathrm{cov}}}^{\dot{*}}\right.$ and $\mathrm{S}_{\text {perf }}^{\dot{ }}$ ) essentially differ, see Figure $4(\mathrm{e})$ and $4(\mathrm{f})$ Alongside the disagreement on the quality indices, $\mathrm{S}_{\mathrm{cov}} \dot{-}$ tends to identify fine clusterings, i.e., 33 clusters on the average,while $\mathrm{S}_{\text {perf }}^{-}$finds clusterings with a coarse granularity, i.e., 2.9 clusters on the average. The absolute variants exhibit a surprisingly similar behavior to the initial clustering with respect to the quality

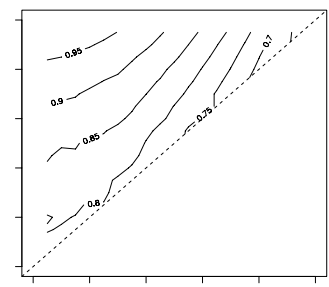

(a) generated clustering

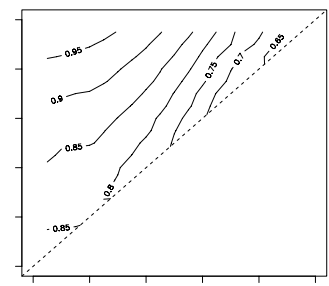

(d) $\mathrm{S}_{\text {perf }}^{-}$-greedy approach

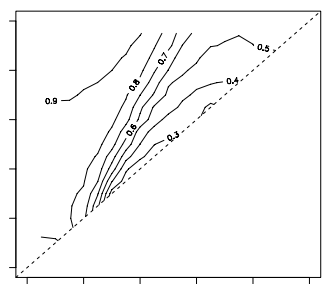

(b) algorithm MCL

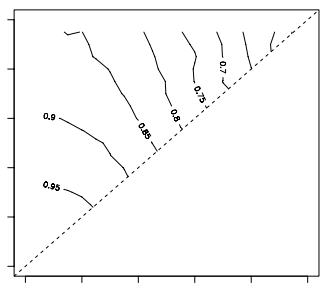

(e) $\stackrel{\dot{\text { cov }}}{\div}$ greedy approach

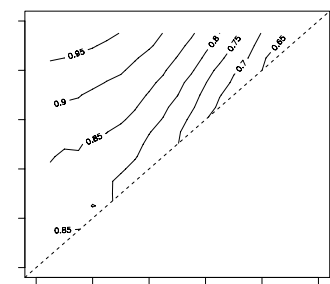

(c) $\mathrm{S}_{\mathrm{cov}}^{-}$-greedy approach

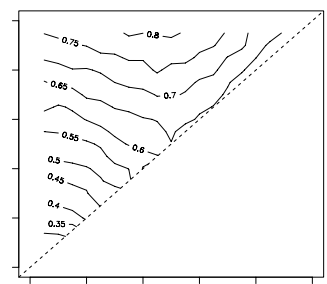

(f) $\mathrm{S}_{\text {perf }}^{\div}$-greedy approach

Fig. 4. Results showing the achieved performance. Probability $p_{\text {in }}$ is shown on the $y$-axis, the $x$-axis holds $p_{\text {out }}$. 


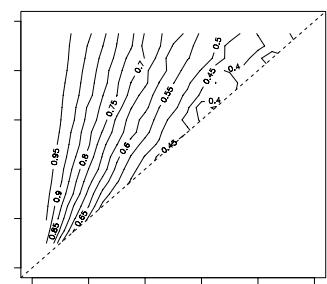

(a) generated clustering

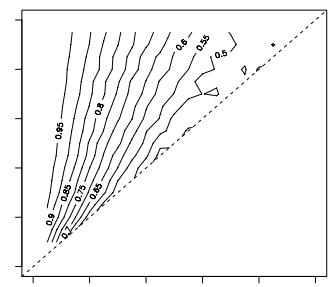

(d) $\mathrm{S}_{\text {perf }}^{-}$-greedy approach

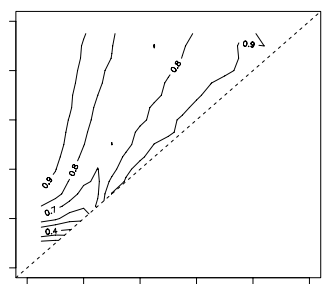

(b) algorithm MCL

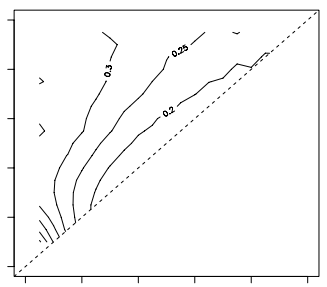

(e) $S_{\text {cov }}^{\div}$-greedy approach

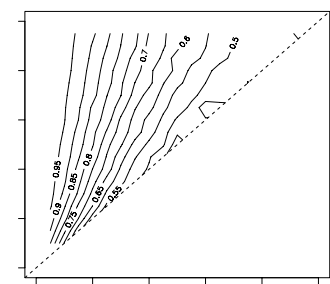

(c) $\mathrm{S}_{\mathrm{cov}}^{-}$-greedy approach

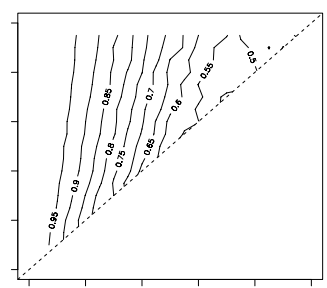

(f) $\mathrm{S}_{\text {perf }}^{\div}$-greedy approach

Fig. 5. Results showing the achieved coverage. Probability $p_{\text {in }}$ is shown on the $y$-axis, the $x$-axis holds $p_{\text {out }}$.

indices. The same holds for $\mathrm{S}_{\text {perf }}^{\div}$with respect to coverage and inter-cluster conductance, however, the behavior is different for performance, but still acceptable scores are attained. In contrast, $\mathrm{S}_{\text {cov }}^{\div}$clearly fails to achieve high values of coverage and inter-cluster conductance, while its performance score is surprisingly good. As observed in [21], the benchmark algorithms do not substantially surpass the initial clustering in general. The same observation holds for the realizations of significance.

In an overall assessment of the achieved clustering quality, the two absolute variants excel with respect to performance for almost all generated instances, with a small advantage of $\mathrm{S}_{\text {perf }}^{-}$over $\mathrm{S}_{\text {cov }}^{-}$(standard modularity). This is particularly meaningful since both do not yield an inappropriately high number of clusters, which would artificially increase performance. With respect to coverage, the absolute variants are only surpassed by the few algorithms that produced a substantially coarser clustering, among those $\mathrm{S}_{\text {perf }}^{\dot{*}}$. An interesting observation is that, using the significance measures as quality indices themselves, all four greedy algorithms attain the maximum corresponding score for most testsets. However, in the case of $A_{\text {strong }}$, the obtained differences in the significance measures are small among most algorithms.

Explaining Some Artifacts. The high values of performance, attained by $\mathrm{S}_{\text {cov }} \dot{-}$ for $A_{\text {sparse }}$ are due to the fact that the large number of clusters identified by this algorithm yields a large fraction of non-connected pairs of nodes that are 
in different clusters. In turn, $\mathrm{S}_{\text {cov }}^{\dot{*}}$ producing fine clusterings can be explained as follows. Each step of the algorithm increases coverage and $\mathbb{E}[$ coverage $]$, which are both bounded by 1 . These values increase faster, if an already large cluster is further enlarged. Thus, the fraction tends to 1 for coarse clusterings, causing $\mathrm{S}_{\mathrm{cov}}^{\circ}$ to terminate early.

Real Data. We have applied our algorithms to a number of realworld networks, due to limited space we only present the most prominent one. Figure[ [shows how the variants of significance perform on the karate club network, studied initially by Zachary [22. The network represents friendship between the 34 members of a university club that, due to an internal dispute, split up into two groups (circular nodes on the left and square-shaped nodes on the right). Clearly, relative performance significance $\left(\mathrm{S}_{\mathrm{perf}}^{\div}\right)$excels here, misclassifying only a single rather ambiguous node (10) and surpassing even modularity in precision.

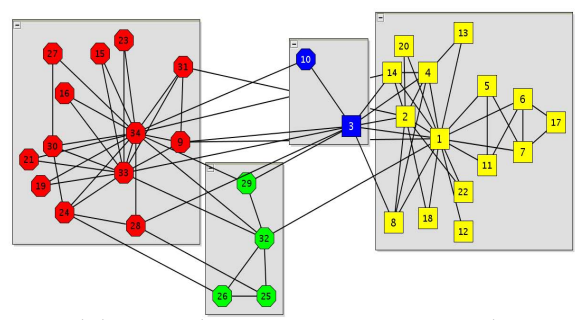

(a) $\mathrm{S}_{\text {cov }}^{-}$(weighted modularity)

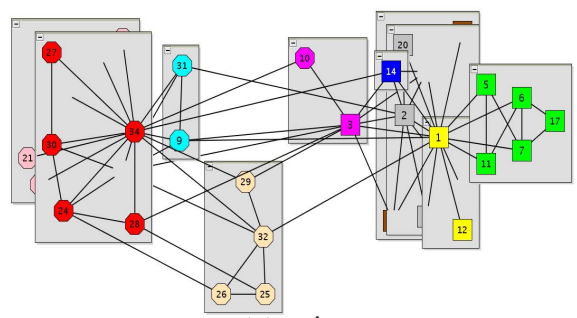

(c) $S_{\text {cov }}^{\div}$

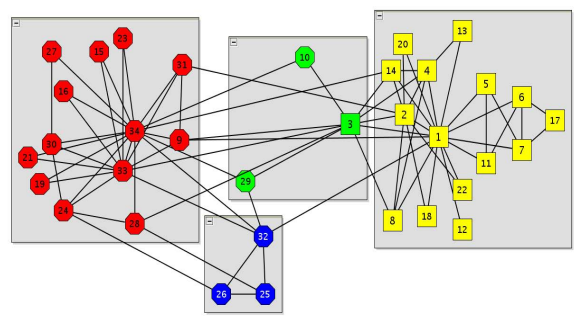

(b) $\mathrm{S}_{\text {perf }}^{-}$

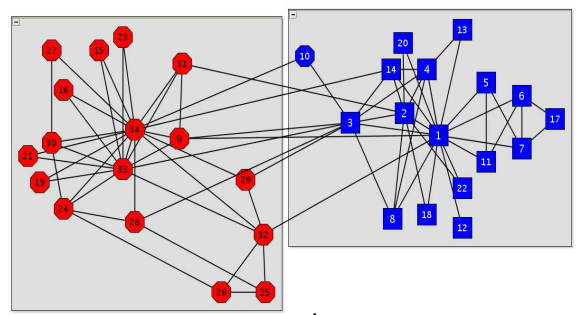

(d) $S_{\text {perf }}^{\div}$

Fig. 6. The results of the greedy significance algorithms (groupings) on Zachary's karate club 22] are in agreement with our experimental evaluation. The variants $\mathrm{S}_{\text {cov }}^{-}$ and $\mathrm{S}_{\text {perf }}^{-}$produce almost the same clustering. While both clusterings are meaningful and close to the real grouping (node shapes), relative performance significance $\left(\mathrm{S}_{\mathrm{perf}}^{\div}\right)$ yields a bisection which is almost exactly the real grouping. Obviously, $\mathrm{S}_{\mathrm{cov}}^{\div}$fails to find a reasonable clustering for this network.

Figure 7 shows an anonymized graph of the email contacts at our department over a period of three months (approx. 44300 emails). Nodes represent persons and weighted edges represent the number of email contacts between two coworkers. The grouping depicts the department's internal structure while the node 
colors (gray values) show the findings of community structure of the greedy algorithm based on $\mathrm{S}_{\text {perf }}^{-}$. Since this example is based on the intuition that the graph structure reflects the grouping, we cleaned the network of artifact nodes with no links to other nodes in its reference cluster (approx. $7.5 \%$ of the original nodes).

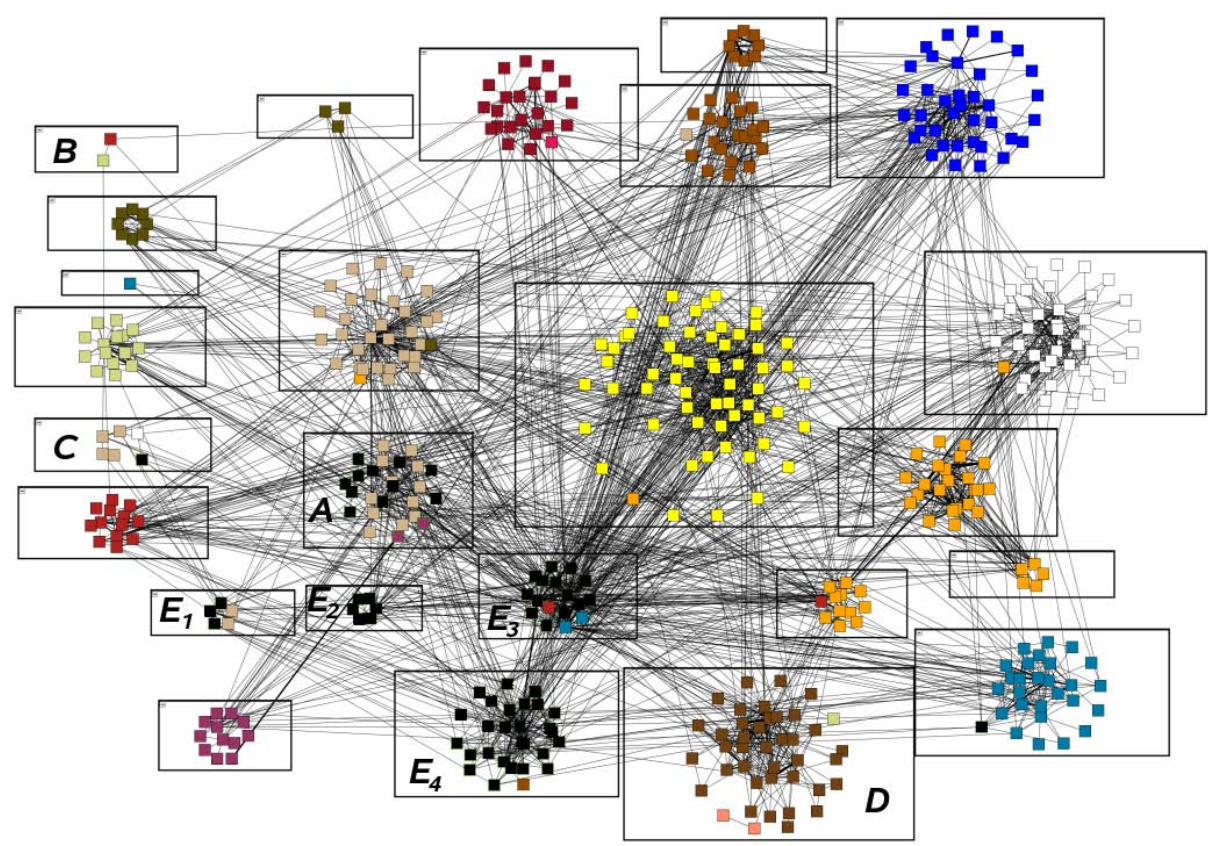

Fig. 7. A network of email contacts at our department. The grouping depicts the department's internal structure as a reference, and the node colors (gray values) are the community detection result of absolute performance significance $\left(\mathrm{S}_{\text {perf }}^{-}\right)$. Inside reference clusters, $\mathrm{S}_{\text {perf }}^{-}$misclassifies only $6.8 \%$ of nodes, most of which are due to the highly ambiguous reference cluster $A$, which is split in half by the algorithm. The clustering of $\mathrm{S}_{\text {perf }}^{-}$yields a noticeably higher $(\approx 6 \%)$ coverage, which is partly due to 9 clusters each being merged into other clusters they are strongly connected with. In terms of inter-cluster conductance and all four realizations of significance, $\mathrm{S}_{\text {perf }}^{-}$ slightly surpasses the reference. However, the performance of the reference clustering is approx. $2.4 \%$ higher than that found by $\mathrm{S}_{\text {perf }}^{-}$. On the whole, a closer investigation explains most disagreements between the two clusterings, e.g., note the artifact nodes in clusters $B, C, D$ and the strong connections between clusters $A, E_{1}, \ldots, E_{4}$, which account for the aggregation done by the algorithm.

\section{Conclusion}

Based on modularity [2], a recently introduced quality measure for graph clusterings, we formally stated a new clustering paradigm, significance, that considers the trade-off between the achieved quality and the expected quality with respect 
to networks with a similar intrinsic structure. The performed experimental study is a systematic evaluation of this paradigm and a substantial advance towards a generalized understanding of the concept modularity is based upon. Summarizing, the evaluation yields that the significance paradigm is highly feasible for several realizations, producing clusterings with remarkable quality. Moreover, the generality of our approach is corroborated by the good results of $\mathrm{S}_{\text {perf }}^{\div}$on real networks and by the fact that there is a general algorithmic approach for many relative realizations, as presented in Algorithm 2. We suggest $\mathrm{S}_{\text {perf }} \dot{\text { as a }}$ strong community detection algorithm if a low or constant number of clusters is expected, such as coarsely structured social networks. Moreover, $\mathrm{S}_{\text {perf }}^{-}$offers a promising alternative to standard modularity, since it yields clusterings of equaling quality, yet it is based on the more appropriate quality index performance.

\section{References}

1. Gaertler, M.: Clustering. In Brandes, U., Erlebach, T., eds.: Network Analysis: Methodological Foundations. Volume 3418 of Lecture Notes in Computer Science. Springer-Verlag (2005) 178-215

2. Newman, M.E.J., Girvan, M.: Finding and evaluating community structure in networks. Phys. Rev. E 69 (2004)

3. Newman, M.E.J.: A fast algorithm for detecting community structure in networks. Technical report, Department of Physics and Center for the Study of Complex Systems, University of Michigan (2003)

4. Fortunato, S., Barthelemy, M.: Resolution Limit in Community Detection. arXiv.org physics/0607100 (2006)

5. Ziv, E., Middendorf, M., Wiggins, C.: Information-Theoretic Approach to Network Modularity. Phys. Rev. E 71 (2005)

6. Muff, S., Rao, F., Caflisch, A.: Local Modularity Measure for Network Clusterizations. Phys. Rev. E 72 (2005)

7. Fine, P., Paolo, E.D., Philippides, A.: Spatially Constrained Networks and the Evolution of Modular Control Systems. In: 9th Intl. Conference on the Simulation of Adaptive Behavior (SAB). (2006)

8. Newman, M.E.J.: Fast Algorithm for Detecting Community Structure in Networks. Physical Review E 69 (2004)

9. Clauset, A., Newman, M.E.J., Moore, C.: Finding community structure in very large networks. Phys. Rev. E 70 (2004)

10. Newman, M.: Modularity and Community Structure in Networks. In: Proceedings of the National Academy of Sciences. (2005) 8577-8582

11. White, S., Smyth, P.: A Spectral Clustering Approach to Finding Communities in Graph. In: SIAM Data Mining Conference. (2005)

12. Guimerà, R., Sales-Pardo, M., Amaral, L.A.N.: Modularity from Fluctuations in Random Graphs and Complex Networks. Physical Review E 70 (2004)

13. Reichardt, J., Bornholdt, S.: Statistical Mechanics of Community Detection. arXiv.org cond-mat/0603718 (2006)

14. Duch, J., Arenas, A.: Community Detection in Complex Networks using Extremal Optimization. Physical Review E 72 (2005)

15. Brandes, U., Delling, D., Gaertler, M., Görke, R., Hoefer, M., Nikoloski, Z., Wagner, D.: Maximizing modularity is hard, arxiv preprint.http://arxiv.org/ abs/physics/0608255 (2006) 
16. van Dongen, S.M.: Graph Clustering by Flow Simulation. PhD thesis, University of Utrecht (2000)

17. Coffin, M., Saltzmann, M.J.: Statistical analysis of computational tests of algorithms and heuristics. 12 (2000)

18. Newman, M.: Analysis of Weighted Networks. Technical report, Cornell University, Santa Fe Institute, University of Michigan (2004)

19. Clauset, A., Newman, M.E.J., Moore, C.: Finding community structure in very large networks. Technical report, University of New Mexico, University of Michigan (2004)

20. Brodal, G.S., Jacob, R.: Dynamic planar convex hull. In: FOCS. (2002) 617-626

21. Brandes, U., Gaertler, M., Wagner, D.: Experiments on Graph Clustering Algorithms. In: Proceedings of the 11th Annual European Symposium on Algorithms (ESA'03). Volume 2832 of Lecture Notes in Computer Science. (2003) 568-579

22. Zachary, W.: An information flow model for conflict and fission in small groups. Journal of Anthropological Research 33 (1977) 452-473

\section{A Expected performance and the Heuristic}

Lemma 3. If the probability of an edge between nodes $v$ and $w$ is defined to be $\frac{\omega(v) \omega(w)}{(2 W)}$ the expected value of performance is (for unweighted edges set $\omega(e) \equiv 1$ for all edges)

$$
\frac{2 \frac{1}{2 W} \sum_{i}\left(\sum_{v \in C_{i}} \omega(v)\right)^{2}+\omega_{\max }\left(n^{2}-\sum_{i}\left|C_{i}\right|^{2}\right)-2 W}{n(n-1) \omega_{\max }}
$$

Proof.

$$
\begin{aligned}
& \mathbb{E}(\mathrm{p}) \\
& =\frac{\mathbb{E}\left(\sum_{\text {intra-e }} \omega(e)\right)+\mathbb{E}\left(\sum_{\text {inter }-e}\left(\omega_{\max }-\omega(e)\right)\right)+\mathbb{E}\left(\sum_{\text {non-inter }-e} \omega_{\max }\right)}{\frac{1}{2} n(n-1) \omega_{\max }} \\
& =\frac{\sum_{i} \frac{1}{2} \sum_{v \in C_{i}} \sum_{w \in C_{i}} 2 W \frac{\omega(v) \omega(w)}{(2 W)^{2}}+\sum_{i} \frac{1}{2} \sum_{v \in C_{i}} \sum_{w \notin C_{i}}\left(\omega_{\max }-2 W \frac{\omega(v) \omega(w)}{(2 W)^{2}}\right)}{\frac{1}{2} n(n-1) \omega_{\max }} \\
& =\frac{\frac{1}{2 W} \sum_{i}\left(\sum_{v \in C_{i}} \omega(v)\right)^{2}+\sum_{i} \sum_{v \in C_{i}} \sum_{w \notin C_{i}}\left(\omega_{\max }\right)}{n(n-1) \omega_{\max }} \\
& \quad-\frac{\frac{1}{2 W} \sum_{i} \sum_{v \in C_{i}} \sum_{w \notin C_{i}}(\omega(v) \omega(w))}{n(n-1) \omega_{\max }} \\
& =\frac{2 \frac{1}{2 W} \sum_{i}\left(\sum_{v \in C_{i}} \omega(v)\right)^{2}+\omega_{\max }\left(n^{2}-\sum_{i}\left|C_{i}\right|^{2}\right)-2 W}{n(n-1) \omega_{\max }}
\end{aligned}
$$

Lemma 4. The heuristic of setting the expected weight of an edge between nodes $v$ and $w$ to $\frac{\omega(v) \omega(w)}{(2 W)}$ does not lead to an expected total edge weight of $W$. 
Proof.

$$
\begin{aligned}
& \sum_{\substack{\{v, w\} \\
v, w \in V}} \frac{\omega(v) \cdot \omega(w)}{2 W}=\frac{1}{2}\left(\sum_{\substack{(v, w) \\
v, w \in V}} \frac{\omega(v) \cdot \omega(w)}{2 W}+\sum_{v \in V} \frac{(\omega(v))^{2}}{2 W}\right) \\
& =\frac{1}{4 W}\left(\sum_{v \in V} \omega(v)\right)^{2}+\frac{1}{4 W} \sum_{v \in V}(\omega(v))^{2}=W+\underbrace{\frac{1}{4 W} \sum_{v \in V}(\omega(v))^{2}}_{>0 \text { since } W \neq 0} \neq W
\end{aligned}
$$

Thus, the expected sum of edge weights does not equal $W$.

Note that if we do not allow self-loops we obtain a similar result. However, since in most real-world graphs the maximum node degree is clearly sublinear in the total sum of edge weights, the relative error, caused by the abovementioned edge probabilities, tends to zero. This can be observed in Equation [6. 